\title{
The polytopal structure of the tight-span of a totally split-decomposable metric
}

\author{
K. T. Huber ${ }^{\mathrm{c}}$, J. Koolen ${ }^{\mathrm{b}}$, V.Moulton ${ }^{\mathrm{c}, *}$ \\ ${ }^{a}$ School of Computing Sciences, University of East Anglia, Norwich, UK \\ ${ }^{b}$ Wen-Tsun Wu Key Laboratory of CAS, School of Mathematical Sciences, \\ University of Science and Technology of China, Hefei, Anhui, 230026, P.R. China. \\ ${ }^{c}$ School of Computing Sciences, University of East Anglia, Norwich, UK
}

\begin{abstract}
The tight-span of a finite metric space is a polytopal complex that has appeared in several areas of mathematics. In this paper we determine the polytopal structure of the tight-span of a totally split-decomposable (finite) metric. These metrics are a generalization of tree-metrics and have importance within phylogenetics. In previous work, we showed that the cells of the tight-span of such a metric are zonotopes that are polytope isomorphic to either hypercubes or rhombic dodecahedra. Here, we extend these results and show that the tight-span of a totally split-decomposable metric can be broken up into a canonical collection of polytopal complexes whose polytopal structures can be directly determined from the metric. This allows us to also completely determine the polytopal structure of the tight-span of a totally split-decomposable metric. We anticipate that our improved understanding of this structure may lead to improved techniques for phylogenetic inference.
\end{abstract}

Keywords: tight-span, totally split-decomposable metric, Buneman graph, Buneman complex

${ }^{*}$ Corresponding author 


\section{Introduction}

In this paper, $X$ will denote a finite set with $|X| \geq 2$. Given a metric $d$ on $X$, the tight-span of $d$ is the polytopal complex $T(d)$ which consists of the bounded faces of the polyhedron

$$
\left\{f \in \mathbb{R}^{X}: f(x)+f(y) \geq d(x, y), \text { for all } x, y \in X\right\}
$$

The tight-span of an arbitrary metric was first introduced by Isbell [22] (where it was called the injective hull), and was subsequently redisovered in [5, 7]. It has appeared in various areas of mathematics including group theory [8, 24], phylogenetics [3, 15], network flow theory [18, tropical geometry [6] and the theory of low distortion embeddings [2].

In this paper we are interested in determining the polytopal structure of the tight-span of a special type of metric on a finite set called a totally splitdecomposable metric [3. We picture the 1-skeleton for an example of the tightspan of such a metric in Figure 1. This type of metric originated from the study of tree-metrics arising in evolutionary biology, and is now commonly used in the phylogenetic analysis of molecular DNA sequence data [21. Recently, infinite versions of these metrics have also appeared in the study of certain finitely generated groups [25].

The tight-span of a totally split-decomposable metric has an interesting polytopal structure. In [1] it was shown that the tight-span of a totally splitdecomposable metric arising from a bipartite graph is a cubical complex. Then, some of the first results concerning this structure for totally split-decomposable metrics in general appeared in [14, 15]. Amongst other things, in these papers it was shown that the tight-span of a certain subclass of the totally splitdecomposable metrics can be considered as a special type of median complex called the Buneman complex (cf. 4] for more concerning median complexes). Subsequently, in [20] it was shown that the cells in the tight-span (i.e. the polytopes from which the tight-span is comprised) of a totally split-decomposable metric are zonotopes that are polytope isomorphic to either hypercubes or rhom- 
bic dodecahedra. In this paper, we shall extend this result by completely determining the polytopal structure of the tight-span of a totally split-decomposable metric.

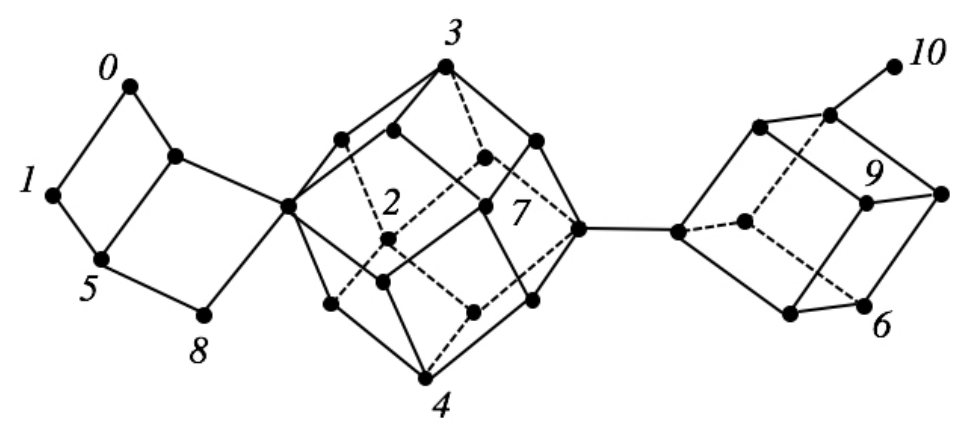

Figure 1: An example of the 1-skeleton of the tight-span of a totally split-decomposable metric defined on the set $\{0,1,2, \ldots, 9,10\}$ (the metric is defined in the next section). It is a polytopal complex consisting of 5 blocks: two 1-cubes, a 3-cube, a rhombic dodecahedron and a pair of 2 -cubes with an edge in common.

We now summarize the rest of the paper. In Section 2 we begin by presenting some terminology and results concerning split systems, structures which form the basis for defining totally split-decomposable metrics. We then present a structural result (cf. Corollary 2) concerning a certain graph that can be associated to a split system (called the incompatibility graph). This result provides a key to breaking up the tight-span of a totally split-decomposable metric into easier to understand pieces.

In Section 3 , we study properties of the Buneman complex, a polytopal complex that can be associated to a weighted split system $(\mathcal{S}, \alpha)$. As we shall explain, we can break this complex down into pieces or blocks (maximal connected subcollections of cells, which cannot be disconnected by removing a vertex or 0-cell) which are in bijective correspondence with the connected components of the incompatibility graph of $\mathcal{S}$. In Section 4 we then prove a result concerning the blocks of the Buneman complex (Theorem 7), which ultimately allows us in Section 7 to reduce the problem of understanding the polytopal structure of the tight-span of a totally split-decomposable metric to that of understanding 
its blocks.

In Section 5 we begin to relate properties of the Buneman complex to those of the tight-span. We do this by considering a map $\kappa$ that maps the Buneman complex onto the tight-span, which was defined in [11 and further studied in [20. In particular, we show that the map $\kappa$ has certain properties relative to the blocks of the Buneman complex, which allows us to prove in Section 6 that the map $\kappa$ induces a bijection between the blocks of the Buneman complex and the blocks of the tight-span (Theorem 15).

In Section 7 we conclude by showing that, for most of the blocks in the Buneman complex, $\kappa$ induces a polytopal complex isomorphism between the blocks in the Buneman complex and their corresponding blocks in the tight-span (Theorem 18). Moreover, we see that the remaining blocks in the tight-span have a very simple structure: they are all rhombic dodecahedra (Theorem 15). As we shall also explain in Section 7, this allows us to completely determine the polytopal structure of the tight-span of a totally split-decomposable metric.

In future work it could be of interest to understand how our results may extend to the case of infinite, totally split-decomposable metrics defined in [25]. In addition, it could be of interest to understand how tight-spans of totally split-decomposable metrics fit the theory of $\operatorname{CAT}(0)$ complexes [4. Ultimately, we anticipate that better understanding the structure of the tight-span of a totally split-decomposable metric might be useful within phylogenetics. For example, this structure is closely-related to the block-realization of a metric [16, which may give insights on how to decompose metrics that are of importance in phylogenetics.

Throughout the paper we will follow and extend the notation and definitions presented in 20]. For the reader's convenience, we shall briefly recall relevant notation from [20, but we refer the reader to that paper for more detail.

\section{The incompatibility graph of weakly compatible split system}

We begin by recalling some terminology and results concerning split systems, structures which form the basis for defining and understanding totally split- 
decomposable metrics. A split of $X$ is a bipartition of $X$, and a set $\mathcal{S}$ of splits of $X$ is a split system (on $X$ ). We denote a split $\{A, B\}$ of $X$ with $\emptyset \neq A, B \subset X$ by $A \mid B(=B \mid A)$. For a split $S$ of $X$ and some elements $x \in X$ we denote by $S(x)$ the element of $S$ that contains $x$ and by $\bar{S}(x)$ the complement of $S(x)$ in $X$. Two distinct splits $S, S^{\prime} \in \mathcal{S}$ are compatible if there exists $A \in S$ and $A^{\prime} \in S^{\prime}$ such that $A \cup A^{\prime}=X$, otherwise $S$ and $S^{\prime}$ are incompatible. We call a split system $\mathcal{S}$ incompatible if every pair of distinct splits in $\mathcal{S}$ is incompatible. We also define a split system with 1 element to be incompatible. Following [3], we call a split system weakly compatible if there exist no three splits $S_{1}, S_{2}, S_{3} \in \mathcal{S}$ and four elements $x_{0}, x_{1}, x_{2}, x_{3} \in X$ such that

$$
S_{j}\left(x_{i}\right)=S_{j}\left(x_{0}\right) \text { if and only if } i=j .
$$

Note that in [13] weakly compatible split systems where characterized as those split system $\mathcal{S}$ for which for any three splits $S_{1}, S_{2}, S_{3} \in \mathcal{S}$ and all $x \in X$, we have $\overline{S_{1}}(x) \cap \overline{S_{2}}(x) \cap \overline{S_{3}}(x) \in\left\{\overline{S_{1}}(x) \cap \overline{S_{2}}(x), \overline{S_{2}}(x) \cap \overline{S_{3}}(x), \overline{S_{1}}(x) \cap \overline{S_{3}}(x)\right\}$. Also note that in [13, Theorem 4.1], the following result is proven. Suppose that $\mathcal{S}=\left\{S_{1}, \ldots, S_{k}\right\}$ is a weakly compatible yet incompatible split system of size $1 \leq k$. Then there exists a partition $X=X_{1} \dot{\cup} \ldots \dot{\cup} X_{2 k}$ of $X$ into $2 k$ non-empty pairwise disjoint subsets $X_{i}$ such that either $\mathcal{S}$ is strictly circular, that is $S_{i}=X_{i} \dot{\cup} \ldots \dot{\cup} X_{i+k-1} \mid X_{i+k} \dot{\cup} \ldots \dot{\cup} X_{i-1}$ holds for $1 \leq i \leq k$ or $\mathcal{S}$ is octahedral, that is, $k=4$ and we can relabel the elements in $\mathcal{S}$ such that $S_{i}=X_{i} \dot{\cup} X_{i+1} \dot{\cup} X_{i+2} \mid X_{i+3} \dot{\cup} X_{i+4} \dot{\cup} X_{i+5}$ for $1 \leq i \leq 3$ and $S_{4}=X_{1} \dot{\cup}$ $X_{3} \dot{\cup} X_{5} \mid X_{2} \dot{\cup} X_{4} \dot{\cup} X_{6}$ (where we take indices modulo $2 k$ ). See Figure 2 for a diagrammatic representation of an octahedral split system. For $\mathcal{S}$ a split system on $X$, let $\operatorname{Oct}(\mathcal{S})=\left\{\mathcal{S}^{\prime} \subseteq \mathcal{S}: \mathcal{S}^{\prime}\right.$ is octahedral $\}$. We call $\mathcal{S}$ consistent if $\mathcal{S}$ is weakly compatible and does not contain an octahedral subsystem, that is, if $\operatorname{Oct}(\mathcal{S})$ is empty.

Using this last result we now present a key property of weakly compatible split systems.

Theorem 1. Suppose that $\mathcal{S} \subsetneq \mathcal{S}(X)$ is a weakly compatible split system and that $\mathcal{S}_{1} \subsetneq \mathcal{S}$ is an octahedral split system. Then every split $S$ in $\mathcal{S}-\mathcal{S}_{1}$ is compatible with every element in $\mathcal{S}_{1}$. 


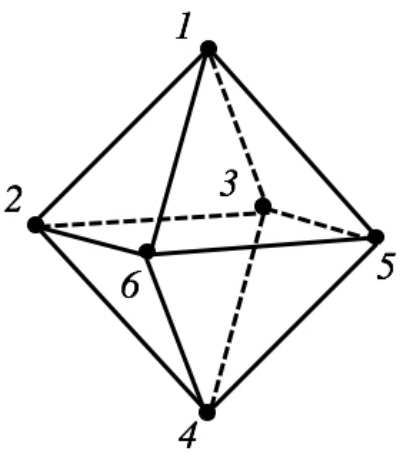

(a)

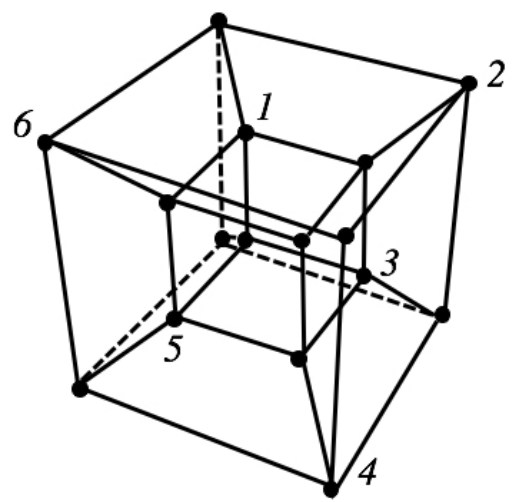

(b)

Figure 2: (a) An octahedral split system on the set $\{1,2,3,4,5,6\}$ consisting of the splits $\{1,2,3\}|\{4,5,6\},\{2,3,4\}|\{5,6,1\},\{3,4,5\} \mid\{6,1,2\}$, and $\{1,3,5\} \mid\{2,4,6\}$, in which each split is obtained by taking the labels of some face and its opposite face in the pictured octahedron.

(b) The Buneman complex associated to the split system in (a).

Proof: Suppose $\mathcal{S}_{1}=\left\{S_{1}, S_{2}, S_{3}, S_{4}\right\}$ and assume for contradiction that there exists some split $S_{5} \in \mathcal{S}-\mathcal{S}_{1}$ such that $S_{5}$ is incompatible with some split in $\mathcal{S}_{1}$. Then

$$
\mathcal{S}^{\prime}=\left\{S \in \mathcal{S}_{1}: S_{5} \text { and } S \text { are incompatible }\right\} \neq \emptyset .
$$

Assume without loss of generality that $S_{i}=X_{i} \dot{\cup} X_{i+1} \dot{\cup} X_{i+2} \mid X_{i+3} \dot{\cup} X_{i+4} \dot{\cup} X_{i+5}$, $i=1,2,3$ and $S_{4}=X_{1} \dot{\cup} X_{3} \dot{\cup} X_{5} \mid X_{2} \dot{\cup} X_{4} \dot{\cup} X_{6}$ for $X=X_{1} \dot{\cup} \ldots \dot{U} X_{6}$.

If $\left|\mathcal{S}^{\prime}\right|=1$ then we may assume without loss of generality that $\mathcal{S}_{1}=\left\{S_{1}\right\}$. But then $S_{5}$ is compatible with all $S_{i}, i=2,3,4$. Since $\left\{S_{2}, S_{3}, S_{4}\right\}$ is strictly circular, there exists some $x \in X$ such that $S_{5}(x) \subsetneq S_{i}(x), i=2,3,4$. Hence, $S_{5}(x) \subseteq \bigcap_{i=2,3,4} S_{i}(x)=X_{j}$, for some $j \in\{1, \ldots, 6\}$. Thus, $S_{5}$ and $S_{1}$ cannot be incompatible since either $X_{j} \subseteq S_{1}(x)$ or $X_{j} \subseteq \overline{S_{1}}(x)$ holds; a contradiction.

Now suppose $\left|\mathcal{S}^{\prime}\right|=2$ and let $x \in X$. Without loss of generality we may assume $x \in X_{1}$ and $\mathcal{S}_{1}=\left\{S_{1}, S_{2}\right\}$. Hence,

$$
\overline{S_{1}}(x) \cap \overline{S_{2}}(x)=\overline{S_{3}}(x) \cap \overline{S_{4}}(x) .
$$

Since $\left\{S_{1}, S_{2}, S_{5}\right\}$ is weakly compatible and incompatible, there exist $i, j \in$ 
$\{1,2,5\}$ such that

$$
\emptyset \neq \overline{S_{i}}(x) \cap \overline{S_{j}}(x)=\overline{S_{1}}(x) \cap \overline{S_{2}}(x) \cap \overline{S_{5}}(x)=\overline{S_{3}}(x) \cap \overline{S_{4}}(x) \cap \overline{S_{5}}(x) .
$$

and so $\overline{S_{3}}(x) \cap \overline{S_{5}}(x) \neq \emptyset$ and $\overline{S_{4}}(x) \cap \overline{S_{5}}(x) \neq \emptyset$. Since $S_{5}$ and $S_{3}$ are compatible it follows that either $\overline{S_{5}}(x) \cap S_{3}(x)=\emptyset$ or $\overline{S_{3}}(x) \cap S_{5}(x)=\emptyset$ must hold. If $\overline{S_{5}}(x) \cap S_{3}(x)=\emptyset$ then $\overline{S_{5}}(x) \cap S_{4}(x) \neq \emptyset$ as otherwise 2 would imply $\overline{S_{5}}(x) \subseteq$ $\overline{S_{1}}(x)$ which is impossible. Since $S_{5}$ and $S_{4}$ are compatible $\overline{S_{4}}(x) \cap S_{5}(x)=\emptyset$ follows. Hence, $\overline{S_{4}}(x) \subseteq \overline{S_{5}}(x)$. Combined with $\overline{S_{5}}(x) \cap S_{3}(x)=\emptyset$, it follows that $\overline{S_{4}}(x) \subseteq \overline{S_{3}}(x)$ which is impossible. Hence, $\overline{S_{3}}(x) \cap S_{5}(x)=\emptyset$.

Since $S_{5}$ and $S_{4}$ are compatible either $\overline{S_{5}}(x) \cap S_{4}(x)=\emptyset$ or $\overline{S_{4}}(x) \cap S_{5}(x)=\emptyset$ must hold. If $\overline{S_{5}}(x) \cap S_{4}(x)=\emptyset$ held, then $\overline{S_{3}}(x) \subsetneq \overline{S_{5}}(x) \subsetneq \overline{S_{4}}(x)$ which is impossible. Thus $\overline{S_{4}}(x) \cap S_{5}(x)=\emptyset$. But then $S_{5}(x) \subseteq S_{4}(x) \cap S_{3}(x)$. Since $\mathcal{S}_{1}$ is weakly compatible yet incompatible 20 implies

$$
\bigcap_{i=1, \ldots, 4} \overline{S_{i}}(x)=\overline{S_{1}}(x) \cap \overline{S_{2}}(x)=\overline{S_{3}}(x) \cap \overline{S_{4}}(x) .
$$

Hence, by [13, Lemma 2.1]

$$
\bigcap_{i=1, \ldots, 4} S_{i}(x)=S_{1}(x) \cap S_{2}(x)=S_{3}(x) \cap S_{4}(x) .
$$

Consequently, $S_{5}(x) \subseteq S_{1}(x) \cap S_{2}(x)$ and so $S_{5}(x) \subseteq S_{1}(x)$ which is impossible.

If $\left|\mathcal{S}^{\prime}\right|=3$ and $x \in X$, then we may assume without loss of generality that $x \in X_{1}$ and $\mathcal{S}^{\prime}=\left\{S_{1}, S_{2}, S_{3}\right\}$. Hence,

$$
\overline{S_{1}}(x) \cap \overline{S_{2}}(x)=\overline{S_{3}}(x) \cap \overline{S_{4}}(x)
$$

and similar arguments to the case $\left|\mathcal{S}^{\prime}\right|=2$ imply that $\overline{S_{4}}(x) \cap \overline{S_{5}}(x) \neq \emptyset$. Thus, since $S_{4}$ and $S_{5}$ are compatible, either $\overline{S_{4}}(x) \cap S_{5}(x)=\emptyset$ or $\overline{S_{5}}(x) \cap S_{4}(x)=\emptyset$ must hold.

We first consider the case $\overline{S_{4}}(x) \cap S_{5}(x)=\emptyset$. Then $X_{2} \dot{\cup} X_{4} \dot{\cup} X_{6} \subsetneq \overline{S_{5}}(x)$. Hence, there exists some $y \in \overline{S_{5}}(x)-\left(X_{2} \dot{\cup} X_{4} \dot{\cup} X_{6}\right)$. Now, since $X=X_{1} \dot{\cup} \ldots \dot{\cup} X_{6}$ it follows that we can pick some $y \in X_{i}$ for some $i \in\{1,3,5\}$. Note that 
$S_{5}(y)=\overline{S_{5}}(x)$, and also that $X_{3}$ is not a subset of $S_{5}(y)$. Indeed, if $X_{3} \subseteq S_{5}(y)$ held, then $X_{2} \cup \dot{\cup} X_{4} \dot{\cup} X_{6} \subsetneq S_{5}(y)$ would imply that $\overline{S_{2}}(x) \subseteq S_{5}(y)$. Hence, $S_{2}$ and $S_{5}$ would be compatible which is impossible. Similarly, we cannot have $X_{1} \subseteq S_{5}(y)$ since in that case $X_{2} \cup \dot{U} X_{4} \dot{\cup} X_{6} \subsetneq \overline{S_{5}}(x)$ would imply $S_{3}(x) \subseteq \overline{S_{5}}(x)$, and thus $S_{3}$ and $S_{5}$ are compatible which is impossible. It follows that there exists $x_{3} \in X_{3}-S_{5}(y)$ and $x_{1} \in X_{1}-S_{5}(y)$. Let $x_{j} \in X_{j}, j \in\{2,4\}$. Then taking the four elements $x_{1}, x_{2}, x_{3}, x_{4}$ together with the three splits $S_{2}, S_{3}, S_{5} \in \mathcal{S}$ violates Property (1), a contradiction.

Now in case $\overline{S_{5}}(x) \cap S_{4}(x)=\emptyset$, we have $X_{1} \dot{\cup} X_{3} \dot{\cup} X_{5} \subsetneq S_{5}(x)$. Hence, there must exist some $y \in S_{5}(x)-\left(X_{1} \dot{\cup} X_{3} \dot{\cup} X_{5}\right)$. Thus, we can pick some element $y \in X_{i}$ for some $i \in\{2,4,6\}$. Note that $S_{5}(x)=S_{5}(y)$. Arguments similar to the ones used in the case of $\overline{S_{4}}(x) \cap S_{5}(x)=\emptyset$ can now be employed to show that there must exist some $x_{2} \in X_{2}-S_{5}(y)$ and some $x_{6} \in X_{6}-S_{5}(y)$. Let $x_{j} \in X_{j}, j \in\{1,3\}$. Then taking the elements $x_{1}, x_{2}, x_{3}, x_{6}$ and the splits $S_{2}, S_{3}, S_{5}$ violates Property (1), which is again a contradiction. This completes the proof for the case $\left|\mathcal{S}^{\prime}\right|=3$.

If $\left|\mathcal{S}^{\prime}\right|=4$, then $\mathcal{S}^{\prime}=\mathcal{S}_{1}$. Hence, $\mathcal{S}^{\prime} \cup\left\{S_{5}\right\}$ is a weakly compatible yet incompatible split system that contains an octahedral split system. By [13, Theorem 4.1], it follows that $\mathcal{S}^{\prime} \cup\left\{S_{5}\right\}$ is octahedral. But then $\left|\mathcal{S}^{\prime} \cup\left\{S_{5}\right\}\right|=4$ which is impossible.

Given a split system $\mathcal{S}$ we define the incompatibility graph $\mathcal{I}(\mathcal{S})$ associated to $\mathcal{S}$ to be the graph with vertex set $\mathcal{S}$ and edge set consisting of those pairs $\left\{S, S^{\prime}\right\}$ of distinct splits $S, S^{\prime} \in \mathcal{S}$ which are incompatible (cf. e.g. [17]). We also let $C(\mathcal{I}(\mathcal{S}))$ denote the set of connected components of $\mathcal{I}(\mathcal{S})$. To illustrate these definitions, let $\mathcal{S}$ denote the split system on $X=\{0,1, \ldots, 9,10\}$ underpinning the totally split-decomposable metric whose tight-span we picture in Figure 1 Then $\mathcal{I}(\mathcal{S})$ is the graph depicted in Figure 3.

Corollary 2. Suppose that $\mathcal{S}$ is a weakly compatible split system, $\mathcal{S}^{\prime} \subseteq \mathcal{S}$ is an octahedral split system, and $\mathcal{I}(\mathcal{S})$ is connected. Then $\mathcal{S}=\mathcal{S}^{\prime}$. In particular, if 


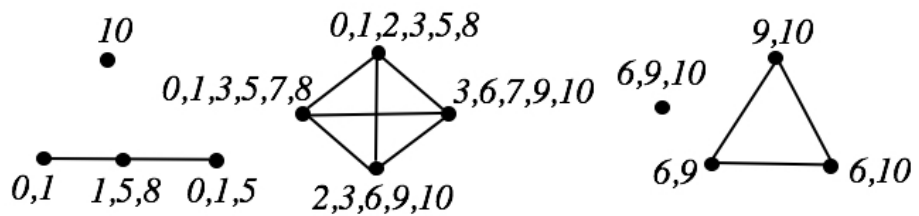

Figure 3: The incompatibility graph of a split system on the set $\{0,1, \ldots, 9,10\}$ underpinning the tight-span pictured in Figure 1 Each vertex corresponds to the split $A \mid \bar{A}$, with $A$ being the set of numbers labelling the vertex (so for, example, the vertex labelled 6, 9,10 corresponds to the split given by taking $\{6,9,10\}$ and its complement).

$\mathcal{S}^{\prime} \in C(\mathcal{I}(\mathcal{S}))$, then $\mathcal{S}^{\prime}$ is octahedral or consistent.

Proof: Suppose to the contrary that $\mathcal{S} \neq \mathcal{S}^{\prime}$. Let $S$ denote a split in $\mathcal{S}-\mathcal{S}^{\prime}$ and let $S^{\prime}$ denote a split in $\mathcal{S}^{\prime}$. Since, by assumption, $\mathcal{I}(\mathcal{S})$ is connected, there exists some path $P$ in $\mathcal{I}(\mathcal{S})$ from $S$ to $S^{\prime}$. But then there must exist some edge $\left\{S_{1}, S_{2}\right\}$ on $P$ such that $S_{1} \in \mathcal{S}-\mathcal{S}^{\prime}$ and $S_{2} \in \mathcal{S}^{\prime}$. But this is impossible since then $S_{1}$ and $S_{2}$ are incompatible in contradiction to Theorem 1 .

\section{The Buneman complex of a weighted split system}

In this section we gather together some useful results concerning the Buneman complex. We begin by presenting some general definitions concerning polyhedral complexes (cf. e.g. [23]).

\subsection{Polytopal complexes}

A polyhedron in $\mathbb{R}^{n}, n \in \mathbb{N}$, is the intersection of a finite collection of halfspaces in $\mathbb{R}^{n}$ and a polytope is a bounded polyhedron. A face of a polyhedron $P$ is the empty-set, $P$ itself, or the intersection of $P$ with a supporting hyperplane. We denote the fact that $P$ is $d$-dimensional by putting $\operatorname{dim}(P)=d$. The 0-dimensional faces of $P$ are also called its vertices and the 1-dimensional faces its edges. A polyhedral complex $\mathcal{C}$ is a finite collection of polyhedra (which we call cells) such that each face of a member of $\mathcal{C}$ is itself a member of $\mathcal{C}$, and the intersection of two members of $\mathcal{C}$ is a face of each. If all members of $\mathcal{C}$ are 
polytopes, we call $\mathcal{C}$ a polytopal complex. The 1-skeleton of $\mathcal{C}$ is the union of its 0- and 1-dimensional cells (which we will also consider sometimes as being a graph, whose vertices and edges correspond to vertices and edges in $\mathcal{C}$ ). Note that we will not usually distinguish between $\mathcal{C}$ and its underlying set $\bigcup_{C \in \mathcal{C}} C$. For any $c$ in the underlying set of $\mathcal{C}$, we let $[c]$ denote the minimal cell $C$ in $\mathcal{C}$ (under cell inclusion) that contains $c$. In this case we also call $c$ a generator of C.

Suppose that $\mathcal{C}$ is a connected polytopal complex. A vertex in $\mathcal{C}$ is a cutvertex if $\mathcal{C}-\{v\}$ is disconnected. Note that if $v$ is a cut-vertex of $\mathcal{C}$ and $C$ is some connected component of $\mathcal{C}-\{v\}$, then $C^{+v}=C \cup\{v\}$ can be regarded as a connected polytopal complex in the obvious way. A maximal collection of cells in $\mathcal{C}$ that is connected and does not contain a cut-vertex is called a block of $\mathcal{C}$. We denote the set of blocks of $\mathcal{C}$ by $\mathcal{B}(\mathcal{C})$. Note that this is also a polytopal complex.

\subsection{The Buneman complex}

A weighted split system $(\mathcal{S}, \alpha)$ (on $X$ ), is a split system $\mathcal{S}$ on $X$ together with a map $\alpha: \mathcal{S} \rightarrow \mathbb{R}^{>0}$. We now define the Buneman complex of such a split system (cf. [20, Section 2.3]). First, we put

$$
\mathcal{U}(\mathcal{S})=\{A \subseteq X: \text { there exists } S \in \mathcal{S} \text { with } A \in S\}
$$

Also, given any map $\phi: \mathcal{U}(\mathcal{S}) \rightarrow \mathbb{R}$, we define

$$
\operatorname{supp}(\phi)=\{A \in \mathcal{U}(\mathcal{S}): \phi(A) \neq 0\}
$$

and put

$$
\mathcal{S}(\phi)=\{S \in \mathcal{S}: S \subseteq \operatorname{supp}(\phi)\} .
$$

Now, we let

$H(\mathcal{S}, \alpha)=\left\{\phi \in \mathbb{R}^{\mathcal{U}(\mathcal{S})}: \phi(A) \geq 0\right.$ and $\phi(A)+\phi(\bar{A})=\frac{\alpha(A \mid \bar{A})}{2}$ for all $\left.A \in \mathcal{U}(\mathcal{S})\right\}$

(a polytope in $\mathbb{R}^{\mathcal{U}(\mathcal{S})}$ which is polytope isomorphic to an $|\mathcal{S}|$-dimensional hypercube) and define the Buneman complex associated to $(\mathcal{S}, \alpha)$ to be the polytopal 
complex

$B(\mathcal{S}, \alpha)=\left\{\phi \in H(\mathcal{S}, \alpha): A_{1}, A_{2} \in \operatorname{supp}(\phi)\right.$ and $\left.A_{1} \cup A_{2}=X \Rightarrow A_{1} \cap A_{2}=\emptyset\right\}$.

Note that $X$ can be considered as a subset of the set of vertices of $B(\mathcal{S}, \alpha)$ via the mapping which takes each $x \in X$ to the map

$$
\phi_{x}: \mathcal{U}(\mathcal{S}) \rightarrow \mathbb{R}^{\geq 0}
$$

given by $\phi_{x}(A)=\alpha(A \mid \bar{A}) / 2$ if $x \notin A$ and 0 otherwise, for all $A \in \mathcal{U}(\mathcal{S})$. For example, for the split system in Figure 2(a) on $X=\{1,2, \ldots, 6\}$ in which all splits are given weight 1, the Buneman complex is the 4-cube in Figure 2(b), where the labelled vertices correspond to the elements in $X$.

We now present two simple observations concerning the Buneman complex that will be useful later on.

Lemma 3. Suppose that $(\mathcal{S}, \alpha)$ is a weighted split system on $X, \phi \in B(\mathcal{S}, \alpha)$ and $\operatorname{dim}([\phi])>0$. Then for all $S \notin \mathcal{S}(\phi)$ and all vertices $\tilde{\phi}$ of $[\phi]$, we have $\left.\tilde{\phi}\right|_{S}=\left.\phi\right|_{S}$.

Proof: This follows from the definition of $B(\mathcal{S}, \alpha)$ (see also [20, Lemma 3.1(i)]).

Lemma 4. Suppose that $(\mathcal{S}, \alpha)$ is a weighted split system on $X$, and $\phi \in$ $B(\mathcal{S}, \alpha)$. Then any pair of distinct splits in $\mathcal{S}(\phi)$ is incompatible.

Proof: If not, then there exist $S_{1}=A_{1}\left|B_{1} \neq S_{2}=A_{2}\right| B_{2}$ in $\mathcal{S}(\phi)$ with $A_{1} \cap A_{2}=\emptyset$, say. So $\overline{A_{1}} \cup \overline{A_{2}}=X$. Since $\overline{A_{1}}, \overline{A_{2}} \in \operatorname{supp}(\phi)$, we obtain $\overline{A_{1}} \cap \overline{A_{2}}=\emptyset$ as $\phi \in B(\mathcal{S}, \alpha)$. Hence, $A_{2}=\overline{A_{1}}$, which contradicts $S_{1} \neq S_{2}$.

\subsection{The blocks of the Buneman complex}

We now study the set $\mathcal{B}(\mathcal{S}, \alpha)$ of blocks of the Buneman complex. In what follows, we consider the 1-skeleton $G(\mathcal{S}, \alpha)$ of $B(\mathcal{S}, \alpha)$ also as being a weighted graph (where, in case an edge corresponds to a split $S \in \mathcal{S}$ in the natural way, it is weighted by $\alpha(S))$. This graph is also known as the (weighted) Buneman graph of $(\mathcal{S}, \alpha)$ (cf. e.g. [10]), and we shall exploit some of its well-known properties. 
In particular, note that each block of the Buneman complex $B(\mathcal{S}, \alpha)$ corresponds to the union of all of those cells in $B(\mathcal{S}, \alpha)$ whose 1-skeleta are contained in some block (i.e. maximal 2-connected component) of $G(\mathcal{S}, \alpha)$. Hence by [17, Theorem 5.1] (which gives a 1-1 correspondence between the blocks of the Buneman graph of a split system $\mathcal{S}$ and the set $C(\mathcal{I}(\mathcal{S}))$ of connected components of $\mathcal{I}(\mathcal{S})$ ), each block in $\mathcal{B}(\mathcal{S}, \alpha)$ corresponds to precisely one element $\mathcal{S}^{\prime} \in C(\mathcal{I}(\mathcal{S})$ ) and, in this case, is isomorphic as a polytopal complex to $B\left(\mathcal{S}^{\prime},\left.\alpha\right|_{\mathcal{S}^{\prime}}\right)$. We shall denote the block of $B(\mathcal{S}, \alpha)$ corresponding to $\mathcal{S}^{\prime} \in C(\mathcal{I}(\mathcal{S})$ ) (considered as a subpolytopal complex of $B(\mathcal{S}, \alpha))$ by $B_{\mathcal{S}^{\prime}}(\mathcal{S}, \alpha)$. In particular, it follows that

$$
\mathcal{B}(\mathcal{S}, \alpha)=\left\{B_{\mathcal{S}^{\prime}}(\mathcal{S}, \alpha): \mathcal{S}^{\prime} \in C(\mathcal{I}(\mathcal{S}))\right\}
$$

Associating to any cell $C$ of $B(\mathcal{S}, \alpha)$ the split system $\mathcal{S}(C)$ induced by $C$ by deleting parallel edges of $C$ and to any collection $\mathcal{C}$ of cells in $B(\mathcal{S}, \alpha)$ the split system $\mathcal{S}(\mathcal{C})=\bigcup_{C \in \mathcal{C}} \mathcal{S}(C)$, we obtain $\mathcal{S}\left(B_{\mathcal{S}^{\prime}}(\mathcal{S}, \alpha)\right)=\mathcal{S}^{\prime}$

We close this section by presenting a connection between the blocks of the Buneman complex of a weighted split system $(\mathcal{S}, \alpha)$ and the incompatibility graph of $\mathcal{S}$.

Lemma 5. Suppose that $(\mathcal{S}, \alpha)$ is a weighted split system on $X$ and $\mathcal{S}^{\prime} \in$ $C(\mathcal{I}(\mathcal{S}))$.

(i) If $\phi \in B_{\mathcal{S}^{\prime}}(\mathcal{S}, \alpha)$ and $S \in \mathcal{S}-\mathcal{S}^{\prime}$, then for all $A \in S$, $\phi(A) \in\{0, \alpha(S) / 2\}$.

(ii) If $\phi_{1} \in B_{\mathcal{S}^{\prime}}(\mathcal{S}, \alpha)$ and $\phi_{2} \in B(\mathcal{S}, \alpha)-B_{\mathcal{S}^{\prime}}(\mathcal{S}, \alpha)$, then there exists a split $S \in \mathcal{S}-\mathcal{S}^{\prime}$ and some $A \in S$ such that $\phi_{1}(A)=0$ and $\phi_{2}(A) \neq 0$.

Proof: (i): Since $\phi$ must be contained in a cell of $B_{\mathcal{S}^{\prime}}(\mathcal{S}, \alpha)$ this is an immediate consequence of [20, Lemma 3.1(i)].

(ii): We first consider the case where $\phi_{2}$ is not a vertex of $B(\mathcal{S}, \alpha)$. Then there exists some split $S=A \mid B \in \mathcal{S}-\mathcal{S}^{\prime}$ such that $\phi_{2}(A)=\alpha$ and $\phi_{2}(B)=\frac{\alpha(S)}{2}-\alpha$ where $0<\alpha<\frac{\alpha(S)}{2}$. But, by (i), $\phi_{1}(A) \in\left\{0, \frac{\alpha(S)}{2}\right\}$ for all $A \in S$. Hence, without loss of generality, $\phi_{1}(A)=0$ and $\phi_{2}(A) \neq 0$.

Now, suppose that $\phi_{2}$ is a vertex of $B(\mathcal{S}, \alpha)$. Then as $\phi_{2} \in B(\mathcal{S}, \alpha)-$ $B_{\mathcal{S}^{\prime}}(\mathcal{S}, \alpha)$ there is a path $\phi, \psi_{1}, \psi_{2}, \ldots, \psi_{m}=\phi_{2}, m \geq 1$, in the 1 -skeleton of $B(\mathcal{S}, \alpha)$ where $\phi$ is a cut-vertex of $B(\mathcal{S}, \alpha)$ contained in $B_{\mathcal{S}^{\prime}}(\mathcal{S}, \alpha)$ such that $\phi_{2}$ is in one of the connected components of $B(\mathcal{S}, \alpha)-\{\phi\}$. Let $S=A \mid B$ be the 
split corresponding to the 1-cell in $B(\mathcal{S}, \alpha)$ with end vertices $\psi_{m-1}$ and $\phi_{2}$. By Lemma 5(i), $\left.\phi_{1}\right|_{S}=\left.\phi\right|_{S}$ follows. Using the isomorphism between the 0-cells of $B(\mathcal{S}, \alpha)$ and the vertices of the Buneman graph $G(\mathcal{S}, \alpha)$ given in 9 , Corollary $3.2]$ let $\phi^{\prime}, \phi_{2}^{\prime}$ denote the vertices in $G(\mathcal{S}, \alpha)$ corresponding to the vertices $\phi, \phi_{2}$, respectively. Without loss of generality, $\phi_{2}^{\prime}(S)=B$. Hence $\phi_{2}(B)=0$ and $\phi_{2}(A)=\alpha(S) / 2$ in view of that isomorphism. By the choice of $S, \phi^{\prime}(S)=A$ follows, and so $\phi(A)=0$ using again that isomorphism. By the choice of $S$, it follows that $0=\phi(A)=\phi_{1}(A)$.

\section{A key result on the blocks of the Buneman complex}

In this section we will prove a key result which, for a weighted split system $(\mathcal{S}, \alpha)$, allows us to decide whether or not two maps $\phi_{1}, \phi_{2} \in B(\mathcal{S}, \alpha)$, are contained within the same block of $B(\mathcal{S}, \alpha)$.

We begin with a lemma concerning cut-vertices of the Buneman complex.

Lemma 6. Suppose that $(\mathcal{S}, \alpha)$ is a weighted split system on $X, \phi$ is a cutvertex of $B(\mathcal{S}, \alpha)$, and $C$ is some connected componen $\hat{1}^{1}$ of $B(\mathcal{S}, \alpha)-\{\phi\}$.

(i) If $S \in \mathcal{S}\left(C^{+\phi}\right)$, and $\phi_{1}, \phi_{2} \in B(\mathcal{S}, \alpha)-C$, then $\left.\phi_{1}\right|_{S}=\left.\phi_{2}\right|_{S}$.

(ii) If $\phi^{\prime} \in C$ then there exists some $S \in \mathcal{S}\left(C^{+\phi}\right)$ such that $\left.\phi^{\prime}\right|_{S} \neq\left.\phi\right|_{S}$.

Proof: (i): In view of Lemma 3 , it suffices to show that $\left.\tilde{\phi}_{1}\right|_{S}=\left.\tilde{\phi}_{2}\right|_{S}$ holds for any pair of vertices $\tilde{\phi}_{1}$ of $\left[\phi_{1}\right]$ and $\tilde{\phi}_{2}$ of $\left[\phi_{2}\right]$. But for any such pair $\tilde{\phi}_{1}$ and $\tilde{\phi}_{2}$, we have that $\tilde{\phi}_{1}$ and $\tilde{\phi}_{2}$ are connected by a shortest path in the 1-skeleton of $B(\mathcal{S}, \alpha)-C$. As, by [9, Corollary 3.2], the 1-skeleton of $B(\mathcal{S}, \alpha)$ is isomorphic to the Buneman graph $G\left(\mathcal{S}^{\prime}, \alpha\right)$ of $(\mathcal{S}, \alpha)$ and for any two vertices $\psi$ and $\psi^{\prime}$ in $G(\mathcal{S}, \alpha)$ we have that $\psi(S)=\psi\left(S^{\prime}\right)$ for all $S^{\prime} \in \mathcal{S}$ not induced by an edge on a shortest path from $\psi$ to $\psi^{\prime}$, it is straight-forward to check that $\left.\tilde{\phi}_{1}\right|_{S}=\left.\tilde{\phi}_{2}\right|_{S}$.

(ii): If $\phi^{\prime}$ is a vertex in $B(\mathcal{S}, \alpha)$, then we can take any split $S \in \mathcal{S}$ induced by some edge on a shortest path in the 1-skeleton of $B(\mathcal{S}, \alpha)$ between $\phi$ and $\phi^{\prime}$. So assume that $\operatorname{dim}\left(\left[\phi^{\prime}\right]\right) \geq 1$. Let $\phi_{1}$ denote a vertex in $\left[\phi^{\prime}\right]$ such that the

\footnotetext{
${ }^{1}$ Here we are using connected component in the topological sense - see e.g. [26, p.103]
} 
number $m$ of edges on a path $P$ from $\phi_{1}$ to $\phi$ in the 1-skeleton of $B(\mathcal{S}, \alpha)$ is as small as possible. If $m=0$ then $\phi_{1}=\phi$ and, so, there must exist some $S \in \mathcal{S}\left(\phi^{\prime}\right) \subseteq \mathcal{S}\left(C^{+\phi}\right)$ such that $\left.\phi^{\prime}\right|_{S} \neq\left.\phi\right|_{S}$ as $\phi^{\prime}$ is not a vertex in $B(\mathcal{S}, \alpha)$ whereas $\phi$ is.

If $m \neq 0$ then there must exist some edge $e$ on $P$ such that for the split $S_{e} \in \mathcal{S}$ induced by $e$ we have $\left.\phi_{1}\right|_{S_{e}} \neq\left.\phi\right|_{S_{e}}$. Note that $S_{e} \in \mathcal{S}\left(C^{+\phi}\right)$, as $\phi_{1} \in C$. Since, by the choice of $\phi_{1}$ we have $\left.\phi^{\prime}\right|_{S_{e}}=\left.\phi_{1}\right|_{S_{e}}$, (ii) follows in this case as well.

We now state and prove the main result of this section. For $\phi_{1} \neq \phi_{2} \in$ $B(\mathcal{S}, \alpha)$, let

$$
\Delta\left(\phi_{1}, \phi_{2}\right)=\left\{S \in \mathcal{S}:\left.\phi_{1}\right|_{S} \neq\left.\phi_{2}\right|_{S}\right\} .
$$

In addition, for $\phi \in B(\mathcal{S}, \alpha)$, note that

$$
\mathcal{S}(\phi)=\{A \mid B \in \mathcal{S}: \phi(A), \phi(B) \neq \emptyset\}=\{S \in \mathcal{S}: S \subseteq \operatorname{supp}(\phi)\} .
$$

Theorem 7. Suppose that $(\mathcal{S}, \alpha)$ is a weighted split system on $X, \phi_{1} \neq \phi_{2} \in$ $B(\mathcal{S}, \alpha)$, and $\mathcal{S}^{\prime} \in C(\mathcal{I}(\mathcal{S}))$. Then $\left\{\phi_{1}, \phi_{2}\right\} \subseteq B_{\mathcal{S}^{\prime}}(\mathcal{S}, \alpha)$ if and only if $\Delta\left(\phi_{1}, \phi_{2}\right) \subseteq$ $\mathcal{S}^{\prime}$.

Proof: First note that without loss of generality we can assume $|C(\mathcal{I}(\mathcal{S}))| \geq 2$, since otherwise $\mathcal{S}=\mathcal{S}^{\prime}$ and $B(\mathcal{S}, \alpha)=B_{\mathcal{S}^{\prime}}(\mathcal{S}, \alpha)$ and so the theorem clearly holds.

Assume for contradiction that $\Delta\left\{\phi_{1}, \phi_{2}\right\} \subseteq \mathcal{S}^{\prime}$ but $\left\{\phi_{1}, \phi_{2}\right\} \not \subset B_{\mathcal{S}^{\prime}}(\mathcal{S}, \alpha)$. It suffices to consider the following two cases:

Case 1: There is a cut-vertex $\phi$ of $B(\mathcal{S}, \alpha)$ so that $\phi_{1}$ and $\phi_{2}$ are both contained in $B(\mathcal{S}, \alpha)-C$ for $C$ the connected component of $B(\mathcal{S}, \alpha)-\{\phi\}$ with $B_{\mathcal{S}^{\prime}}(\mathcal{S}, \alpha) \subseteq$ $C^{+\phi}$. Then since $\mathcal{S}^{\prime} \subseteq \mathcal{S}\left(C^{+\phi}\right)$, Lemma 6(i) implies for all $S \in \mathcal{S}^{\prime}$ that $\left.\phi_{1}\right|_{S}=$ $\left.\phi_{2}\right|_{S}$. Hence $\Delta\left(\phi_{1}, \phi_{2}\right)$ is not a subset of $\mathcal{S}^{\prime}$, a contradiction.

Case 2: There is a cut vertex $\phi$ of $B(\mathcal{S}, \alpha)$ so that $\phi_{1}$ is in a connected component $C$ of $B(\mathcal{S}, \alpha)-\{\phi\}$ with $B_{\mathcal{S}^{\prime}}(\mathcal{S}, \alpha)$ not a subset of $C^{+\phi}$, and $\phi_{2}$ is contained in $D^{+\phi}$ for $D$ the connected component of $B(\mathcal{S}, \alpha)-\{\phi\}$ with $B_{\mathcal{S}^{\prime}}(\mathcal{S}, \alpha) \subseteq D^{+\phi}$. If $\phi_{1} \neq \phi$ then, by Lemma 6(ii), there exists some $S \in \mathcal{S}\left(C^{+\phi}\right)$ such that $\left.\phi_{1}\right|_{S} \neq\left.\phi\right|_{S}$. Moreover, as $S \in \mathcal{S}\left(C^{+\phi}\right)$ we have $S \notin \mathcal{S}^{\prime}$ as $\mathcal{S}\left(C^{+\phi}\right) \cap \mathcal{S}\left(D^{+\phi}\right)=\emptyset$. 
Hence, $S \notin \Delta\left(\phi_{1}, \phi_{2}\right)$. But this is impossible since, by Lemma 6 $6(\mathrm{i}),\left.\phi\right|_{S}=\left.\phi_{2}\right|_{S}$, and so $\left.\phi_{1}\right|_{S} \neq\left.\phi_{2}\right|_{S}$.

So assume $\phi_{1}=\phi$. By Lemma 6(ii), it follows that there must exist some $S \in \mathcal{S}\left(D^{+\phi}\right)$ such that $\left.\phi_{1}\right|_{S} \neq\left.\phi_{2}\right|_{S}$. Hence, $S \in \Delta\left(\phi_{1}, \phi_{2}\right) \subseteq \mathcal{S}^{\prime}$. But this is impossible since $\mathcal{S}^{\prime} \subseteq \mathcal{S}\left(C^{+\phi_{1}}\right)$ and $\mathcal{S}\left(C^{+\phi_{1}}\right) \cap \mathcal{S}\left(D^{+\phi_{1}}\right)=\emptyset$.

Conversely, suppose $\left\{\phi_{1}, \phi_{2}\right\} \subseteq B_{\mathcal{S}^{\prime}}(\mathcal{S}, \alpha)$. Let $S \in \mathcal{S}-\mathcal{S}^{\prime}$. Then there is some cut-vertex $\phi$ of $B(\mathcal{S}, \alpha)$ and some connected component $C$ of $B(\mathcal{S}, \alpha)-\{\phi\}$ with $S \in \mathcal{S}\left(C^{+\phi}\right)$ and $\phi_{1}, \phi_{2} \in B(\mathcal{S}, \alpha)-C$. Hence by Lemma 6 (i), $\left.\phi_{1}\right|_{S}=\left.\phi_{2}\right|_{S}$, i.e. $S \notin \Delta\left(\phi_{1}, \phi_{2}\right)$.

\section{The $\kappa$ map}

In this section, we begin to relate properties of the Buneman complex and the tight-span of a totally split-decomposable metric.

First, recall that a metric $d$ on $X$ is totally split-decomposable if there exists a weighted weakly compatible split system $(\mathcal{S}, \alpha)$ on $X$ with

$$
d=d_{\mathcal{S}, \alpha}=\sum_{S \in \mathcal{S}} \alpha(S) \delta_{S}
$$

where, for any split $S$ of $X$ and all $x, y \in X, \delta_{S}(x, y)=1$ if $S(x) \neq S(y)$ and $\delta_{S}(x, y)=0$ else. If $d$ is such a metric, then it follows by results in 3 , that if $d=d_{\mathcal{S}^{\prime}, \alpha^{\prime}}$ for some weakly compatible split system $\mathcal{S}^{\prime}$ and some weighting $\alpha^{\prime}$ on $\mathcal{S}^{\prime}$, then $\mathcal{S}=\mathcal{S}^{\prime}$ and $\alpha=\alpha^{\prime}$. Thus, in what follows we are interested in determining the polytopal structure of $T\left(d_{\mathcal{S}, \alpha}\right)$ for $(\mathcal{S}, \alpha)$ some weighted weakly compatible split system. For this, we will exploit properties of a certain map $\kappa$ from the Buneman complex $B(\mathcal{S}, \alpha)$ to the tight-span $T\left(d_{\mathcal{S}, \alpha}\right)$ which is defined as follows.

Given a weighted split system $(\mathcal{S}, \alpha)$ on $X$, we define the map

$$
\kappa: \mathbb{R}^{\mathcal{U}(\mathcal{S})} \rightarrow \mathbb{R}^{X}: \phi \mapsto\left(X \rightarrow \mathbb{R}: x \mapsto d_{1}\left(\phi, \phi_{x}\right)\right),
$$


where $d_{1}$ denotes the metric on $\mathbb{R}^{\mathcal{U}(\mathcal{S})}$ defined by setting, for $\phi, \phi^{\prime} \in \mathbb{R}^{\mathcal{U}(\mathcal{S})}$,

$$
d_{1}\left(\phi, \phi^{\prime}\right)=\sum_{A \in \mathcal{U}(\mathcal{S})}\left|\phi(A)-\phi^{\prime}(A)\right| .
$$

Note that $\kappa(B(\mathcal{S}, \alpha))=T\left(d_{\mathcal{S}, \alpha}\right)$ if and only if $\mathcal{S}$ is weakly compatible [1]. Moreover, in case $\mathcal{S}$ is weakly compatible, the map $\kappa^{\prime}$ defined by taking any maximal cell $C$ in $B(\mathcal{S}, \alpha)$ to the cell $[\kappa(\phi)]$, where $\phi$ is any generator of $C$, is a well-defined map which induces a bijection between the maximal cells of $B(\mathcal{S}, \alpha)$ and the maximal cells of $T\left(d_{\mathcal{S}, \alpha}\right)$ [20, Theorem 6.1]

We now prove a useful observation (which is essentially also shown in the proof of (i) $\Rightarrow$ (ii) in [15, Theorem 7.1]).

Theorem 8. Suppose that $(\mathcal{S}, \alpha)$ is a weighted weakly compatible split system on $X$, and $\phi_{1}, \phi_{2} \in B(\mathcal{S}, \alpha)$ distinct with $\kappa\left(\phi_{1}\right)=\kappa\left(\phi_{2}\right)$. If $\phi=\left(\phi_{1}+\phi_{2}\right) / 2$, then $\mathcal{S}(\phi)$ is an octahedral split system.

Proof: As $H(\mathcal{S}, \alpha)$ is convex, $\phi \in H(\mathcal{S}, \alpha)$. As $\kappa$ is linear, $\kappa(\phi)=\kappa\left(\left(\phi_{1}+\right.\right.$ $\left.\left.\phi_{2}\right) / 2\right)=\left(\kappa\left(\phi_{1}\right)+\kappa\left(\phi_{2}\right)\right) / 2$, which is in $T\left(d_{\mathcal{S}, \alpha}\right)$. Therefore $\phi \in B(\mathcal{S}, \alpha)$ (as $\kappa$ maps $B(\mathcal{S}, \alpha)$ surjectively onto $T\left(d_{\mathcal{S}, \alpha}\right)$ and $B(\mathcal{S}, \alpha)=\kappa^{-1}\left(T\left(d_{\mathcal{S}, \alpha}\right)\right) \cap H(\mathcal{S}, \alpha)$ since $\mathcal{S}$ is weakly compatible - see e. g. [11, p. 305]). Put $\mathcal{S}^{\prime}=\mathcal{S}-\mathcal{S}(\phi)$, $\mathcal{S}^{\prime \prime}=\mathcal{S}(\phi), \kappa^{*}=\left.\kappa\right|_{\mathbb{R}^{U}\left(\mathcal{S}^{\prime}\right)}$, and $\kappa^{\prime}=\left.\kappa\right|_{\mathbb{R}^{\mathcal{U}}\left(\mathcal{S}^{\prime \prime}\right)}$.

Note that, as $\phi_{1}, \phi_{2}$ distinct, $\emptyset \neq \Delta\left(\phi_{1}, \phi_{2}\right) \subseteq \mathcal{S}^{\prime \prime}$. So, by Lemma $4, \mathcal{S}^{\prime \prime}$ is incompatible. Thus $B\left(\mathcal{S}^{\prime \prime},\left.\alpha\right|_{\mathcal{S}^{\prime \prime}}\right)=H\left(\mathcal{S}^{\prime \prime},\left.\alpha\right|_{\mathcal{S}^{\prime \prime}}\right)$, by [10, Proposition 3.3].

Moreover, $\phi_{1}^{\prime}=\left.\phi_{1}\right|_{\mathcal{U}\left(\mathcal{S}^{\prime \prime}\right)}, \phi_{2}^{\prime}=\left.\phi_{2}\right|_{\mathcal{U}\left(\mathcal{S}^{\prime \prime}\right)} \in B\left(\mathcal{S}^{\prime \prime},\left.\alpha\right|_{\mathcal{S}^{\prime \prime}}\right)$, and $\phi_{1}^{\prime} \neq \phi_{2}^{\prime}$ since $\Delta\left(\phi_{1}, \phi_{2}\right) \subseteq \mathcal{S}^{\prime \prime}$. But these considerations imply

$$
\kappa^{\prime}\left(\phi_{1}^{\prime}\right)=\kappa\left(\phi_{1}\right)-\kappa^{*}\left(\left.\phi_{1}\right|_{\mathcal{U}\left(\mathcal{S}^{\prime}\right)}\right)=\kappa\left(\phi_{2}\right)-\kappa^{*}\left(\phi_{2} \mid \mathcal{U}\left(\mathcal{S}^{\prime}\right)\right)=\kappa^{\prime}\left(\phi_{2}^{\prime}\right)
$$

since, by [20, Lemma 3.1(i)], $\left.\phi_{1}\right|_{\mathcal{U}\left(\mathcal{S}^{\prime}\right)}=\left.\phi_{2}\right|_{\mathcal{U}\left(\mathcal{S}^{\prime}\right)}$ holds. Thus, $\kappa^{\prime}$ is not injective. Hence $\mathcal{S}^{\prime \prime}$ is not strictly circular by [15, Proposition 5.1].

Since $\mathcal{S}^{\prime \prime}$ is weakly compatible, $\mathcal{S}^{\prime \prime}$ is either strictly circular or octahedral, and so $\mathcal{S}^{\prime \prime}$ is octahedral.

Using this last result we now prove two results which relate properties of $\kappa$ to the blocks of the Buneman complex. The first result shows that $\kappa$ is injective 
when restricted to any block of the Buneman complex that does not correspond to an octahedral split system.

Theorem 9. Suppose that $(\mathcal{S}, \alpha)$ is a weighted weakly compatible split system on $X$ and that $\mathcal{S}^{\prime} \in C(\mathcal{I}(\mathcal{S}))$ is not octahedral, then $\left.\kappa\right|_{B_{\mathcal{S}^{\prime}}(\mathcal{S}, \alpha)}$ is injective.

Proof: Suppose $\phi_{1} \neq \phi_{2} \in B_{\mathcal{S}^{\prime}}(\mathcal{S}, \alpha)$ with $\kappa\left(\phi_{1}\right)=\kappa\left(\phi_{2}\right)$. By Theorem 7 . $\emptyset \neq \Delta\left(\phi_{1}, \phi_{2}\right) \subseteq \mathcal{S}^{\prime}$. Put $\phi=\left(\phi_{1}+\phi_{2}\right) / 2$. By Theorem $8, \mathcal{S}(\phi)$ is an octahedral split system. Since $\mathcal{S}^{\prime}$ is not octahedral we cannot have $\mathcal{S}(\phi) \subseteq \mathcal{S}^{\prime}$, by Corollary 2. Hence, $\Delta\left(\phi_{1}, \phi_{2}\right) \subseteq \mathcal{S}(\phi) \cap S^{\prime}=\emptyset$ which is impossible.

The second result shows that if $\phi_{1}, \phi_{2} \in B(\mathcal{S}, \alpha)$ are distinct and not in the same block of $B(\mathcal{S}, \alpha)$, then their images under $\kappa$ are distinct.

Theorem 10. Suppose that $(\mathcal{S}, \alpha)$ is a weighted weakly compatible split system on $X$. If $\phi_{1}, \phi_{2} \in B(\mathcal{S}, \alpha)$ are distinct, and $\left\{\phi_{1}, \phi_{2}\right\} \nsubseteq B_{\mathcal{S}^{\prime}}(\mathcal{S}, \alpha)$, for any $\mathcal{S}^{\prime} \in C(\mathcal{I}(\mathcal{S}))$, then $\kappa\left(\phi_{1}\right) \neq \kappa\left(\phi_{2}\right)$.

Proof: Suppose for contradiction that $\kappa\left(\phi_{1}\right)=\kappa\left(\phi_{2}\right)$. Let $\phi=\left(\phi_{1}+\phi_{2}\right) / 2$. By Theorem $8 \mathcal{S}(\phi)$ is an octahedral split system. Since $\mathcal{S}(\phi) \subseteq \mathcal{S}^{\prime}$ must hold for some $\mathcal{S}^{\prime} \in C(\mathcal{I}(\mathcal{S}))$, Corollary 2 implies $\mathcal{S}^{\prime}=\mathcal{S}(\phi)$. Thus, $\Delta\left(\phi_{1}, \phi_{2}\right) \subseteq \mathcal{S}^{\prime}$ and so, by Theorem $7,\left\{\phi_{1}, \phi_{2}\right\} \subseteq B_{\mathcal{S}^{\prime}}(\mathcal{S}, \alpha)$, a contradiction.

\section{Relating blocks in $B(\mathcal{S}, \alpha)$ to blocks in $T\left(d_{\mathcal{S}, \alpha}\right)$}

In this section we use the map $\kappa$ to provide an explicit bijection between the blocks of the Buneman complex and the tight-span for the metric $d_{\mathcal{S}, \alpha}$ associated to a weighted weakly compatible split system $(\mathcal{S}, \alpha)$.

We begin by proving that $\kappa$ maps the underlying set of any maximal cell in the Buneman complex onto the underlying set of some maximal cell in the tight-span.

Lemma 11. Suppose that $(\mathcal{S}, \alpha)$ is a weighted weakly compatible split system on $X,[\phi]$ is a maximal cell in $B(\mathcal{S}, \alpha)$ with generator $\phi \in B(\mathcal{S}, \alpha)$, and $\kappa^{\prime}([\phi])=C$, with $C$ a maximal cell in $T\left(d_{\mathcal{S}, \alpha}\right)$. Then $\kappa([\phi])=C$. 
Proof: First note that by the definition of $\kappa^{\prime}, \kappa(\phi)$ is a generator for $C$, i.e. $C=[\kappa(\phi)]$.

Now, suppose $\psi \in[\phi]$, then by [20, Theorem 5.1(iii)] $\kappa(\psi) \in[\kappa(\phi)]=C$. Hence $\kappa([\phi]) \subseteq C$.

Conversely, suppose $f \in C$. Since $\kappa$ is surjective, there is some $\psi \in B(\mathcal{S}, \alpha)$ with $\kappa(\psi)=f$. But as $\kappa(\psi) \in[\kappa(\phi)]$, by [20, Theorem 5.1(iii)], $\psi \in[\phi]$. Hence $f \in \kappa([\phi])$, and so $C \subseteq \kappa([\phi])$.

We now show that the image under $\kappa^{\prime}$ of any pair of maximal cells in the Buneman complex can intersect in at most one point.

Lemma 12. Suppose that $(\mathcal{S}, \alpha)$ is a weighted weakly compatible split system on $X, \mathcal{S}^{\prime}, \mathcal{S}^{\prime \prime} \in C(\mathcal{I}(\mathcal{S}))$ distinct, and that $\Omega, \Omega^{\prime}$ are maximal cells in $B_{\mathcal{S}^{\prime}}(\mathcal{S}, \alpha)$ and $B_{\mathcal{S}^{\prime \prime}}(\mathcal{S}, \alpha)$, respectively. Then $\left|\kappa^{\prime}(\Omega) \cap \kappa^{\prime}\left(\Omega^{\prime}\right)\right| \leq 1$.

Proof: Let $C=\kappa^{\prime}(\Omega)$ and $C^{\prime}=\kappa^{\prime}\left(\Omega^{\prime}\right)$ and, for the purposes of obtaining a contradiction, that $\left|C \cap C^{\prime}\right|>1$. Then as $C \cap C^{\prime}$ is a cell in $T\left(d_{\mathcal{S}, \alpha}\right), \operatorname{dim}(C \cap$ $\left.C^{\prime}\right) \geq 1$.

Now, suppose that $g, g^{\prime}$ are distinct generators for $C \cap C^{\prime}$, which must exist since $\operatorname{dim}\left(C \cap C^{\prime}\right) \geq 1$. Since $\kappa(\Omega)=C$ and $\kappa\left(\Omega^{\prime}\right)=C^{\prime}$ and, by [11, Theorem], $\kappa$ is surjective there must exist $\phi, \phi^{\prime} \in \Omega$ distinct with $\kappa(\phi)=g$ and $\kappa\left(\phi^{\prime}\right)=g^{\prime}$, and $\phi^{\prime \prime}, \phi^{\prime \prime \prime} \in \Omega^{\prime}$ distinct with $\kappa\left(\phi^{\prime \prime}\right)=g$ and $\kappa\left(\phi^{\prime \prime \prime}\right)=g^{\prime}$.

Note that $\left|\Omega \cap \Omega^{\prime}\right| \leq 1$ since $\Omega, \Omega^{\prime}$ are contained in distinct blocks of $B(\mathcal{S}, \alpha)$. If $\left|\Omega \cap \Omega^{\prime}\right|=0$, then we obtain a contradiction to Theorem 10 , since $\kappa(\phi)=g=\kappa\left(\phi^{\prime \prime}\right)$ and $\left\{\phi, \phi^{\prime \prime}\right\}$ is not contained in any block of $B(\mathcal{S}, \alpha)$. Moreover, if $\left|\Omega \cap \Omega^{\prime}\right|=1$, then at least one of $\phi \neq \phi^{\prime \prime}$ and $\phi^{\prime} \neq \phi^{\prime \prime \prime}$ must hold. Without loss of generality we may assume that $\phi \neq \phi^{\prime \prime}$. Then $\left\{\phi, \phi^{\prime \prime}\right\}$ is not contained in any block of $B(\mathcal{S}, \alpha)$. By Theorem 10, $g=\kappa(\phi) \neq \kappa\left(\phi^{\prime \prime}\right)=g$ which is also impossible.

We now show that if a block in the Buneman complex consists of a single maximal cell, then its image under $\kappa$ is the underlying set of some block in the tight-span. 
Proposition 13. Suppose that $(\mathcal{S}, \alpha)$ is a weighted weakly compatible split system on $X, \mathcal{S}^{\prime} \in C(\mathcal{I}(\mathcal{S}))$, and the block $B_{\mathcal{S}^{\prime}}(\mathcal{S}, \alpha)$ consists of a single maximal cell $\Omega$ in $B(\mathcal{S}, \alpha)$. Then $\kappa^{\prime}(\Omega)$ is a block in $T\left(d_{\mathcal{S}, \alpha}\right)$.

Proof: First note that $C=\kappa^{\prime}(\Omega)$ is a maximal cell in $T\left(d_{\mathcal{S}, \alpha}\right)$. Suppose that $C$ is not a block of $T\left(d_{\mathcal{S}, \alpha}\right)$. Then there is some cell $C^{\prime}$ distinct from $C$ in $T\left(d_{\mathcal{S}, \alpha}\right)$ with $C^{\prime} \nsubseteq C$ and $C \cap C^{\prime}$ is a cell with dimension at least 1 . Let $C^{\prime \prime}$ be a maximal cell in $T\left(d_{\mathcal{S}, \alpha}\right)$ containing $C^{\prime}$. Note that $C^{\prime \prime} \neq C$ and $C \cap C^{\prime} \subseteq C \cap C^{\prime \prime}$. Let $\Omega^{\prime} \neq \Omega$ be a maximal cell in $B(\mathcal{S}, \alpha)$ with $\kappa^{\prime}\left(\Omega^{\prime}\right)=C^{\prime \prime}$, which exists since, by [20, Theorem 6.1], $\kappa^{\prime}$ is bijecitve. Note that since $B_{\mathcal{S}^{\prime}}(\mathcal{S}, \alpha)$ contains a single maximal cell, $\Omega^{\prime}$ and $\Omega$ must be contained in different blocks of $B(\mathcal{S}, \alpha)$. Since $\left|\kappa^{\prime}(\Omega) \cap \kappa^{\prime}\left(\Omega^{\prime}\right)\right|>1$, this is impossible in view of Lemma 12 .

We now extend the previous result, and show that the image under $\kappa$ of any block of the Buneman complex is the underlying set of some block in the tight-span.

Theorem 14. Suppose that $(\mathcal{S}, \alpha)$ is a weighted weakly compatible split system on $X$ and $\mathcal{S}^{\prime} \in C(\mathcal{I}(\mathcal{S}))$. Then $\kappa\left(B_{\mathcal{S}^{\prime}}(\mathcal{S}, \alpha)\right)$ is equal to some block of $T\left(d_{\mathcal{S}, \alpha}\right)$.

Proof: By Proposition 13 and Corollary 2, it suffices to assume that $\mathcal{S}^{\prime}$ is consistent and that $B_{\mathcal{S}^{\prime}}(\mathcal{S}, \alpha)$ contains at least 2 maximal cells.

We first show that $\kappa\left(B_{\mathcal{S}^{\prime}}(\mathcal{S}, \alpha)\right)$ is a subset of some block of $T\left(d_{\mathcal{S}, \alpha}\right)$. Suppose that $\Omega, \Omega^{\prime}$ are two distinct maximal cells in $B_{\mathcal{S}^{\prime}}(\mathcal{S}, \alpha)$ with $\operatorname{dim}\left(\Omega \cap \Omega^{\prime}\right) \geq 1$, which must clearly exist as $B_{\mathcal{S}^{\prime}}(\mathcal{S}, \alpha)$ is a block of $B(\mathcal{S}, \alpha)$. We claim that $\operatorname{dim}\left(\kappa^{\prime}(\Omega) \cap \kappa^{\prime}\left(\Omega^{\prime}\right)\right) \geq 1$.

To see that this claim holds, first note that by Lemma 11

$$
\kappa\left(\Omega \cap \Omega^{\prime}\right) \subseteq \kappa(\Omega) \cap \kappa\left(\Omega^{\prime}\right)=\kappa^{\prime}(\Omega) \cap \kappa^{\prime}\left(\Omega^{\prime}\right) .
$$

Hence, $\kappa^{\prime}(\Omega) \cap \kappa^{\prime}\left(\Omega^{\prime}\right) \neq \emptyset$ as $\operatorname{dim}\left(\Omega \cap \Omega^{\prime}\right) \geq 1$ and so $\Omega \cap \Omega^{\prime} \neq \emptyset$. Suppose $\operatorname{dim}\left(\kappa^{\prime}(\Omega) \cap \kappa^{\prime}\left(\Omega^{\prime}\right)\right)<1$. Then $\kappa^{\prime}(\Omega) \cap \kappa^{\prime}\left(\Omega^{\prime}\right)=\{g\}$, with $g$ a vertex of $T\left(d_{\mathcal{S}, \alpha}\right)$. Thus, $\kappa\left(\Omega \cap \Omega^{\prime}\right)=\{g\}$. But, since $\operatorname{dim}\left(\Omega \cap \Omega^{\prime}\right) \geq 1$, there must exist $\phi, \phi^{\prime} \in \Omega \cap$ $\Omega^{\prime} \subseteq B_{\mathcal{S}^{\prime}}(\mathcal{S}, \alpha)$ distinct with $\kappa(\phi)=\kappa\left(\phi^{\prime}\right)=\{g\}$, which contradicts Theorem 9 and therefore proves the claim. 
Containment of $\kappa\left(B_{\mathcal{S}^{\prime}}(\mathcal{S}, \alpha)\right)$ in some block of $T\left(d_{\mathcal{S}, \alpha}\right)$ now follows since, as $B_{\mathcal{S}^{\prime}}(\mathcal{S}, \alpha)$ is a block of $B(\mathcal{S}, \alpha)$, we can find an ordering $\Omega_{1}, \Omega_{2}, \ldots \Omega_{k}, k \geq 2$, of the maximal cells of $B_{\mathcal{S}^{\prime}}(\mathcal{S}, \alpha)$ so that for all $1<i \leq k$, there exists some $1 \leq j<i$ such that $\operatorname{dim}\left(\Omega_{i} \cap \Omega_{j}\right) \geq 1$. Hence by the claim and Lemma 11 it immediately follows that

$$
\kappa\left(B_{\mathcal{S}^{\prime}}(\mathcal{S}, \alpha)\right) \subseteq \bigcup_{i=1}^{k} \kappa\left(\Omega_{i}\right)=\bigcup_{i=1}^{k} \kappa^{\prime}\left(\Omega_{i}\right)
$$

is contained in some block $\mathcal{T}$ of $T\left(d_{\mathcal{S}, \alpha}\right)$.

Now, suppose for contradiction that $\kappa\left(B_{\mathcal{S}^{\prime}}(\mathcal{S}, \alpha)\right)$ is a strictly proper subset of $\mathcal{T}$. Then there must exist two maximal cells $C, C^{\prime}$ in $T\left(d_{\mathcal{S}, \alpha}\right)$ with $\operatorname{dim}\left(C \cap C^{\prime}\right) \geq 1, C \subseteq \kappa\left(B_{\mathcal{S}^{\prime}}(\mathcal{S}, \alpha)\right)$ and $C^{\prime} \subseteq \mathcal{T}-\left(\kappa\left(B_{\mathcal{S}^{\prime}}(\mathcal{S}, \alpha)\right) \cup\left(C \cap C^{\prime}\right)\right)$. Let $\Omega, \Omega^{\prime}$ be distinct maximal cells in $B(\mathcal{S}, \alpha)$ with $\kappa^{\prime}(\Omega)=C$ and $\kappa^{\prime}\left(\Omega^{\prime}\right)=C^{\prime}$. Then $\Omega \subseteq B_{\mathcal{S}^{\prime}}(\mathcal{S}, \alpha)$ (since $C \subseteq \kappa\left(B_{\mathcal{S}^{\prime}}(\mathcal{S}, \alpha)\right)$ ), and $\Omega^{\prime} \subseteq B_{\mathcal{S}^{\prime \prime}}(\mathcal{S}, \alpha)$ with $\mathcal{S}^{\prime \prime} \in C(\mathcal{I}(\mathcal{S}))-\left\{\mathcal{S}^{\prime}\right\}$ (since $C^{\prime} \nsubseteq \kappa\left(B_{\mathcal{S}^{\prime}}(\mathcal{S}, \alpha)\right)$ ). But $\operatorname{dim}\left(C \cap C^{\prime}\right) \geq 1$ and so, in view of the claim, we obtain a contradiction to Lemma 12.

Putting our results together we now show that, by mapping the underlying set of each block of the Buneman complex onto the underlying set of some block in the tight-span, the map $\kappa$ in fact induces a bijection from the blocks of the Buneman complex onto the blocks of the tight-span. Before proving this we recall some definitions and results from [20].

First, for any cell $\Omega$ in the Buneman complex $B(\mathcal{S}, \alpha)$ and any $x \in X$ there exists some map $\gamma^{x} \in \Omega$ called the gate for $x$ in $\Omega$ such that $d_{1}\left(\phi_{x}, \psi\right)=$ $d_{1}\left(\phi_{x}, \gamma^{x}\right)+d_{1}\left(\gamma^{x}, \psi\right)$, for all $\psi \in \Omega$. Second, to any $x \in X$ associate the map $h_{x}$ : $X \rightarrow \mathbb{R}$ in $T\left(d_{\mathcal{S}, \alpha}\right)$ given by $h_{x}(y)=d_{\mathcal{S}, \alpha}(x, y)$, for all $y \in X$. Then, similarly, for any cell $C$ in $T\left(d_{\mathcal{S}, \alpha}\right)$ and any $x \in X$ there exists a (necessarily unique) map $g^{x} \in C$ called the gate of $C$ for $x$ such that $d_{\infty}\left(h_{x}, h\right)=d_{\infty}\left(h_{x}, g^{x}\right)+d_{\infty}\left(g^{x}, h\right)$, for all $h \in C$.

We now prove the aforementioned result.

Theorem 15. Suppose that $(\mathcal{S}, \alpha)$ is a weighted weakly compatible split system 
on $X$. The map

$$
K: \mathcal{B}(B(\mathcal{S}, \alpha)) \rightarrow \mathcal{B}\left(T\left(d_{\mathcal{S}, \alpha}\right)\right): B_{\mathcal{S}^{\prime}}(\mathcal{S}, \alpha) \mapsto \kappa\left(B_{\mathcal{S}^{\prime}}(\mathcal{S}, \alpha)\right)
$$

is a well-defined bijection. Moreover, $K$ induces a bijection between the set of blocks $\left\{B_{\mathcal{S}^{\prime}}(\mathcal{S}, \alpha): \mathcal{S}^{\prime} \in O \operatorname{Oct}(\mathcal{S})\right\}$ in $B(\mathcal{S}, \alpha)$ and the set of cells in $T\left(d_{\mathcal{S}, \alpha}\right)$ that are rhombic dodecahedra. In particular, if a cell in $T\left(d_{\mathcal{S}, \alpha}\right)$ is a rhombic dodecahedron, then it must also be a block of $T\left(d_{\mathcal{S}, \alpha}\right)$.

Proof: By Theorem 14, the map $K$ is well-defined.

To see that $K$ is surjective, suppose $\mathcal{T} \in \mathcal{B}\left(T\left(d_{\mathcal{S}, \alpha}\right)\right)$. Let $C$ be some maximal cell in $\mathcal{T}$. Let $\Omega$ be the maximal cell in $B(\mathcal{S}, \alpha)$ with $\kappa^{\prime}(\Omega)=C$, and let $B_{\mathcal{S}^{\prime}}(\mathcal{S}, \alpha), \mathcal{S}^{\prime} \in C(\mathcal{I}(\mathcal{S}))$, be the block in $B(\mathcal{S}, \alpha)$ containing $\Omega$. Then Lemma 11 combined with Theorem 14 implies $K\left(B_{\mathcal{S}^{\prime}}(\mathcal{S}, \alpha)\right)=\mathcal{T}$.

To see that $K$ is injective, suppose there exist two distinct blocks $B_{\mathcal{S}^{\prime \prime}}(\mathcal{S}, \alpha)$, $B_{\mathcal{S}^{\prime}}(\mathcal{S}, \alpha), \mathcal{S}^{\prime}, \mathcal{S}^{\prime \prime} \in C(\mathcal{I}(\mathcal{S}))$, in $B(\mathcal{S}, \alpha)$ with $K\left(B_{\mathcal{S}^{\prime}}(\mathcal{S}, \alpha)\right)=K\left(B_{\mathcal{S}^{\prime \prime}}(\mathcal{S}, \alpha)\right)$. Then there must exist $f \in K\left(B_{\mathcal{S}^{\prime}}(\mathcal{S}, \alpha)\right), \phi_{1} \in B_{\mathcal{S}^{\prime}}(\mathcal{S}, \alpha)-B_{\mathcal{S}^{\prime \prime}}(\mathcal{S}, \alpha)$ and $\phi_{2} \in B_{\mathcal{S}^{\prime \prime}}(\mathcal{S}, \alpha)-B_{\mathcal{S}^{\prime}}(\mathcal{S}, \alpha)$ with $\kappa\left(\phi_{1}\right)=\kappa\left(\phi_{2}\right)=f$. But this contradicts Theorem 10. Thus, $K$ is a bijection.

Now, let $\mathcal{R}$ denote the set of cells in $T\left(d_{\mathcal{S}, \alpha}\right)$ that are rhombic dodecahedra. Suppose $\mathcal{S}^{\prime} \in \operatorname{Oct}(\mathcal{S})$. Then, by Corollary $2 B_{\mathcal{S}^{\prime}}(\mathcal{S}, \alpha)$ is a block of $B(\mathcal{S}, \alpha)$ consisting of a single cell $\Omega$. Hence, $\Omega$ is a maximal cell of $B(\mathcal{S}, \alpha)$. Let $\omega \in B(\mathcal{S}, \alpha)$ denote a generator for $\Omega$. Then $\mathcal{S}(\omega) \subseteq \mathcal{S}\left(B_{\mathcal{S}^{\prime}}(\mathcal{S}, \alpha)\right)=\mathcal{S}^{\prime}$. Since $\mathcal{S}(\omega)$ is maximal incompatible and $\mathcal{S}^{\prime}$ is incompatible it follows that $\mathcal{S}(\omega)=\mathcal{S}^{\prime}$. Hence, $\mathcal{S}(\omega)$ is octahedral. To see that then $\kappa^{\prime}(\Omega)$ must be a rhombic dodecahedron, we next consider the underlying graph $\varsigma^{2} U G\left(\mathcal{G}\left(\kappa^{\prime}(\Omega)\right), d^{\prime}:=\left.d_{\infty}\right|_{\mathcal{G}\left(\kappa^{\prime}(\Omega)\right)}\right)$ - where $\mathcal{G}\left(\kappa^{\prime}(\Omega)\right)$ is the set of gates of $\kappa^{\prime}(\Omega)$ - and $U G\left(\Gamma(\Omega),\left.d_{1}\right|_{\Gamma(\Omega)}\right)$ - where $\Gamma(\Omega)$ is the set of gates of $\Omega$.

Note first that by the remark directly following the proof of [20, Claim 2, p.476] $d^{\prime}$ is cell-decomposable as $d_{\mathcal{S}, \alpha}$ is totally split-decomposable. By [19, Theorem 1.1], $\left(\mathcal{G}\left(\kappa^{\prime}(\Omega)\right), d^{\prime}\right)$ is a proper antipodal metric space and $T\left(\mathcal{G}\left(\kappa^{\prime}(\Omega)\right), d^{\prime}\right)$ is

\footnotetext{
${ }^{2}$ For any finite metric space $(Y, d)$ the underlying graph, denoted by $U G(Y, d)$, has vertex set $Y$ and edge set consisting of those 2-sets $\{x, y\} \subseteq Y$ for which there exists no $z \in Y-\{x, y\}$ such that $d(x, y)=d(x, z)+d(z, y)$
} 
polytope isomorphic to $\kappa^{\prime}(\Omega)$. Since $\kappa$ induces an isometry between $\left(\mathcal{G}\left(\kappa^{\prime}(\Omega)\right), d^{\prime}\right)$ and $\left(\Gamma(\Omega),\left.d_{1}\right|_{\Gamma(\Omega)}\right)$ the proof of [20, Claim 2, p.476] implies that $U G\left(\Gamma(\Omega),\left.d_{1}\right|_{\Gamma(\Omega)}\right)$ and $U G\left(\mathcal{G}\left(\kappa^{\prime}(\Omega)\right), d^{\prime}\right)$ are isomorphic graphs. By [20, Lemma 3.1], $d_{1}\left(\gamma^{x}, \gamma^{y}\right)=$ $\sum_{S \in \mathcal{S}(\omega)} \alpha(S) \delta_{S}(x, y)$ for all $x, y \in X$. A straight forward check shows that $U G\left(\Gamma(\Omega),\left.d_{1}\right|_{\Gamma(\Omega)}\right)$ is isomorphic to $K_{3 \times 2}$. Thus, $U G\left(\mathcal{G}\left(\kappa^{\prime}(\Omega)\right), d^{\prime}\right)$ is isomorphic to $K_{3 \times 2}$. Hence $T\left(\mathcal{G}\left(\kappa^{\prime}(\Omega)\right), d^{\prime}\right)$ is polytope isomorphic with a rhombic dodecahedron [19, Theorem 4.3] and, so $\kappa^{\prime}(\Omega)$ is polytope isomorphic with a rhombic dodecahedron. Therefore $K\left(\left\{B_{\mathcal{S}^{\prime}}(\mathcal{S}, \alpha): \mathcal{S}^{\prime} \in \operatorname{Oct}(\mathcal{S})\right\}\right) \subseteq \mathcal{R}$.

Conversely, suppose $R$ is a rhombic dodecahedron in $T\left(d_{\mathcal{S}, \alpha}\right)$. Since $T\left(d_{\mathcal{S}, \alpha}\right)$ is a polytopal complex and, by [20, Corollary 7.3] every cell of $T\left(d_{\mathcal{S}, \alpha}\right)$ is polytope isomorphic with either a hypercube or a rhombic dodecahedron, it follows that $R$ must be a maximal cell of $T\left(d_{\mathcal{S}, \alpha}\right)$. Thus, there exists some maximal cell $\Psi$ of $B(\mathcal{S}, \alpha)$ such that $\kappa^{\prime}(\Psi)=R$. Let $\xi \in B(\mathcal{S}, \alpha)$ denote a generator of $\Psi$. Assume for contradiction that $\mathcal{S}(\xi)$ is circular. Then it is straight forward to verify that $U G\left(\Gamma(\Psi),\left.d\right|_{\Gamma(\Psi)}\right)$ is a $2 m$-cycle where $m=|\mathcal{S}(\xi)|$. Since $\left(\Gamma(\Psi),\left.d_{1}\right|_{\Gamma(\Psi)}\right)$ and $\left(\mathcal{G}(R), d^{\prime \prime}\right)$ are isometric metric spaces, where $d^{\prime \prime}=\left.d_{\infty}\right|_{\mathcal{G}(R)}$, it follows that $U G\left(\mathcal{G}(R), d^{\prime \prime}\right)$ is also a $2 m$-cycle. Hence, by [19, Theorem 4.2], $T\left(\mathcal{G}(R), d^{\prime \prime}\right)$ is an $m$-cube. Thus, $R$ is also an $m$-cube which is impossible. Consequently, $\mathcal{S}(\xi)$ must be octahedral. By Corollary $2, \Psi$ is the block $B_{\mathcal{S}(\psi)}(\mathcal{S}, \alpha)$ of $B(\mathcal{S}, \alpha)$.

\section{The polytopal structure of $T\left(d_{\mathcal{S}, \alpha}\right)$}

In this section, we conclude by explaining how to obtain the polytopal structure of the tight-span of a totally split-decomposable metric directly from the Buneman complex (and hence from its underlying split system). We shall do this at the end of the section, but first we need to consider the polytopal structure of the blocks in $T\left(d_{\mathcal{S}, \alpha}\right)$.

First, note that by Theorem 15 each maximal cell $R$ in $T\left(d_{\mathcal{S}, \alpha}\right)$ that is a rhombic dodecahedron is also a block of $T\left(d_{\mathcal{S}, \alpha}\right)$. Moreover, for such a cell, there exists some $\mathcal{S}^{\prime} \in O c t(\mathcal{S})$, such that $\kappa$ maps the underlying set of the block $B_{\mathcal{S}^{\prime}}(\mathcal{S}, \alpha)$ (which is polytope isomorphic to a 4 -cube - see Figure 2 ) onto $R$. In 
particular, $\kappa$ restricted to $B_{\mathcal{S}^{\prime}}(\mathcal{S}, \alpha)$ does not induce a polytope isomorphism between $B_{\mathcal{S}^{\prime}}(\mathcal{S}, \alpha)$ and $R$. We shall now show that (see Theorem 18 below), in contrast to octahedral split systems in $\mathcal{S}$, in case $\mathcal{S}^{\prime} \in C(\mathcal{I}(\mathcal{S}))-\operatorname{Oct}(\mathcal{S})$ (i.e $\mathcal{S}^{\prime}$ is a consistent split system), $\kappa$ actually induces a polytopal complex isomorphism between $B_{\mathcal{S}^{\prime}}(\mathcal{S}, \alpha)$ and the block of $T\left(d_{\mathcal{S}, \alpha}\right)$ that has underlying set $\kappa\left(B_{\mathcal{S}^{\prime}}(\mathcal{S}, \alpha)\right)$. To prove this we shall require some further terminology.

Let $V$ be a finite-dimensional $\mathbb{R}$-vector space and $P \subseteq V$. A subset $T \subseteq P$ is an extremal subset of $P$ if, for any $u, v \in P$, and any positive real numbers $\gamma, \beta>0$ with $\gamma+\beta=1$, the assumption $\gamma u+\beta v \in T$ implies $u, v \in T$ (see [12, p. 51]). For the following proposition we assume $V=\mathbb{R}^{X}$.

Proposition 16. Suppose that $(\mathcal{S}, \alpha)$ is a weighted weakly compatible split system on $X$, and $\mathcal{S}^{\prime} \in C(\mathcal{I}(\mathcal{S}))$. Then $K\left(B_{\mathcal{S}^{\prime}}(\mathcal{S}, \alpha)\right)$ is an extremal subset of $P\left(d_{\mathcal{S}, \alpha}\right)$.

Proof: Let $\mathcal{T}=K\left(B_{\mathcal{S}^{\prime}}(\mathcal{S}, \alpha)\right)=\kappa\left(B_{\mathcal{S}^{\prime}}(\mathcal{S}, \alpha)\right)$. Suppose $f, g \in P\left(d_{\mathcal{S}, \alpha}\right), \gamma, \beta>0$, $\gamma+\beta=1$, and $\gamma f+\beta g \in \mathcal{T}$. We need to show that $f, g \in \mathcal{T}$.

First note that $T\left(d_{\mathcal{S}, \alpha}\right)$ is an extremal subset of $P\left(d_{\mathcal{S}, \alpha}\right)$. Hence $f, g \in$ $T\left(d_{\mathcal{S}, \alpha}\right)$. Moreover, as $\kappa$ maps $B(\mathcal{S}, \alpha)$ onto $T\left(d_{\mathcal{S}, \alpha}\right)$ there exist $\phi, \phi^{\prime} \in B(\mathcal{S}, \alpha)$ with $\kappa(\phi)=f$ and $\kappa\left(\phi^{\prime}\right)=g$. Since $\gamma \phi+\beta \phi^{\prime} \in H(\mathcal{S}, \alpha)$ clearly holds, it follows in view of $B(\mathcal{S}, \alpha)=\kappa^{-1}\left(T\left(d_{\mathcal{S}, \alpha}\right)\right) \cap H(\mathcal{S}, \alpha)$ that $\gamma \phi+\beta \phi^{\prime} \in B(\mathcal{S}, \alpha)$.

Now, as $\kappa$ is linear, we have

$$
\kappa\left(\gamma \phi+\beta \phi^{\prime}\right)=\gamma \kappa(\phi)+\beta \kappa\left(\phi^{\prime}\right)=\gamma f+\beta g \in \mathcal{T} .
$$

Hence, by Theorem 15 we have $\gamma \phi+\beta \phi^{\prime} \in B_{\mathcal{S}^{\prime}}(\mathcal{S}, \alpha)$.

Assume for contradiction that $\left\{\phi, \phi^{\prime}\right\} \nsubseteq B_{\mathcal{S}^{\prime}}(\mathcal{S}, \alpha)$. Without loss of generality $\phi \notin B_{\mathcal{S}^{\prime}}(\mathcal{S}, \alpha)$. Put $\phi_{1}=\gamma \phi+\beta \phi^{\prime} \in B_{\mathcal{S}^{\prime}}(\mathcal{S}, \alpha)$ and $\phi_{2}=\phi \notin B_{\mathcal{S}^{\prime}}(\mathcal{S}, \alpha)$. Then by Lemma 5 (ii), there exists a split $S \in \mathcal{S}$ and some $A \in S$ such that

$$
0=\phi_{1}(A)=\left(\gamma \phi+\beta \phi^{\prime}\right)(A)=\gamma \phi(A)+\beta \phi^{\prime}(A)
$$

and $0 \neq \phi_{2}(A)=\phi(A)$ which is impossible.

Hence $\left\{\phi, \phi^{\prime}\right\} \subseteq B_{\mathcal{S}^{\prime}}(\mathcal{S}, \alpha)$. But then $f=\kappa(\phi), g=\kappa\left(\phi^{\prime}\right) \in \mathcal{T}$, as required. 
To establish the property of the map $\kappa$ mentioned at the beginning of this section, we shall use the following result from [12] which we recall for the convenience of the reader.

Theorem 17. [12, Theorem 1(ii)] Let $V$ and $V^{\prime}$ be finite dimensional real vector spaces, let $P \subseteq V$ and $P^{\prime} \subseteq V^{\prime}$ be convex sets and let $f: V^{\prime} \rightarrow V$ be some affine map with $f\left(P^{\prime}\right) \subseteq P$. Moreover let $T$ be some extremal subset of $P$ and let $x \in T^{\prime}:=f^{-1}(T) \cap P^{\prime}$. If $f$ maps $T^{\prime}$ bijectively into $T$ then $f$ maps the smallest extremal subset of $P^{\prime}$ containing $x^{\prime}$ bijectively onto the smallest extremal subset of $P$ containing $f(x)$.

Theorem 18. Suppose that $(\mathcal{S}, \alpha)$ is a weighted weakly compatible split system on $X$, and $\mathcal{S}^{\prime} \in C(\mathcal{I}(\mathcal{S}))-\operatorname{Oct}(\mathcal{S})$. Then $K\left(B_{\mathcal{S}^{\prime}}(\mathcal{S}, \alpha)\right)$ is isomorphic to $B_{\mathcal{S}^{\prime}}(\mathcal{S}, \alpha)$ as polytopal complexes.

Proof: Let $V^{\prime}=\mathbb{R}^{\mathcal{U}(\mathcal{S})}, V=\mathbb{R}^{X}, P^{\prime}=H(\mathcal{S}, \alpha), P=P\left(d_{\mathcal{S}, \alpha}\right), f=\kappa: V^{\prime} \rightarrow V$, $T=K\left(B_{\mathcal{S}^{\prime}}(\mathcal{S}, \alpha)\right)$, and $T^{\prime}=f^{-1}(T) \cap P^{\prime}$. Then it is straight forward to see that $f\left(P^{\prime}\right) \subseteq P$ and $T^{\prime}=B_{\mathcal{S}^{\prime}}(\mathcal{S}, \alpha)$ hold. Moreover, by Theorem 16, $T$ is an extremal subset of $P$, and by Theorems 15 and $9, f$ maps $T^{\prime}$ bijectively onto $T$. The theorem now follows by applying Theorem 17.

We now explain how to determine the polytopal structure of $T\left(d_{\mathcal{S}, \alpha}\right)$ from the weighted split system $(\mathcal{S}, \alpha)$. By the results above, $T\left(d_{\mathcal{S}, \alpha}\right)$ has one block for each $\mathcal{S}^{\prime} \in C(\mathcal{I}(\mathcal{S})$ ), and each of these blocks is polytope isomorphic to a rhombic dodecahedron in case $\mathcal{S}^{\prime} \in \operatorname{Oct}(\mathcal{S})$ and to the Buneman complex $B\left(\mathcal{S}^{\prime},\left.\alpha\right|_{\mathcal{S}^{\prime}}\right)$ otherwise (for example, consider the weighted split-system $(\mathcal{S}, \alpha)$ with $\alpha$ the all-one weight function whose incompatibility graph is pictured in Figure 3 and whose tight-span $T\left(d_{\mathcal{S}, \alpha}\right)$ is pictured in Figure 1). Moreover, we can obtain $T\left(d_{\mathcal{S}, \alpha}\right)$ as a polytopal complex by starting with the Buneman complex $B\left(\mathcal{S},\left.\alpha\right|_{\mathcal{S}}\right)$ and replacing each 4 -cube in $B\left(\mathcal{S},\left.\alpha\right|_{\mathcal{S}}\right)$ corresponding to an element in $\operatorname{Oct}(\mathcal{S})$ by a rhombic dodecahedron. This is done by first identifying the 6 vertices in the 4-cube within the Buneman complex which can be canonically identified as pictured in Figure 2 Then we remove the 4-cube and replace it with a rhombic dodecahedron in which the 6 vertices now correspond to the vertices labelled $2,3,7,4$ and the cut vertices in the rhombic dodecahedron forming the block in Figure 1). 


\section{Acknowledgements}

The authors thank Andreas Dress for some helpful and interesting discussions concerning octahedral split systems. They also thank the reviewers for their helpful comments. JK was partially supported by the National Natural Science Foundation of China (No. 11471009 and No. 11671376).

\section{References}

[1] H.-J. Bandelt and V. Chepoi, Cellular bipartite graphs, European J Combinatorics, 17, 121-134, (1996)

[2] H.-J. Bandelt and V. Chepoi, Embedding metric spaces in the rectilinear plane: a six-point criterion, Discrete Comput. Geom., 15, 107-117, (1996)

[3] H. J. Bandelt and A. Dress, A canonical decomposition theory for metrics on a finite set, Adv. Math. 92 (1992) 47-105.

[4] V. Chepoi, Graphs of some CAT(0) complexes, Adv. Math. 24 (2000) 125179

[5] M. Chorbak and L. Lamore, Generosity helps or an 11-competitive algorithm for three servers, J. Algorithms 16 (1994), 234-263.

[6] M. Develin, B. Sturmfels, Tropical convexity, Doc. Math., 9, (2004), 1-27

[7] A. Dress, Trees, tight extensions of metric spaces, and the cohomological dimension of certain groups: A note on combinatorial properties of metric spaces, Adv. Math. 53 (1984) 321-402.

[8] A. Dress, Towards a classification of transitive group actions on finite metric space, Adv. Math. 74 (1989) 163-189.

[9] A.Dress, K.T.Huber, and V.Moulton, Some variations on a theme by Buneman, Ann. Combin 1 (1997) 339-352.

[10] A. Dress, M. Hendy, K. T. Huber, and V. Moulton, On the number vertices and edges in the Buneman graph, Ann. Combin. 1 (1997) 329-337. 
[11] A. Dress, K. T. Huber, and V. Moulton, A comparison between two distinct models in projective cluster theory: The median and the tight-span construction, Ann. Combin. 2 (1998) 299-311.

[12] A. Dress, K. T. Huber, and V.Moulton, Affine maps that induce polytopal complex isomorphisms, Discrete Comput. Geom. 24 (2000) 49-60.

[13] A. Dress, K. T. Huber, and V. Moulton, An exceptional split geometry, Ann. Combin. 4 (2000) 1-11.

[14] A. Dress, K. T. Huber, and V. Moulton, Totally split-decomposable metrics of combinatorial dimension two, Ann. Combin. 5 (2001) 97-110.

[15] A. Dress, K. T. Huber, and V. Moulton, An explicit computation of the injective hull of certain finite metric spaces in terms of their associated Buneman complex, Adv. Math. 168 (2002) 1-28.

[16] A. Dress, K. T. Huber, J. Koolen, and V. Moulton, Compatible decompositions and block realizations of metric spaces, European J. Combinatorics, 29 (2008) 1617-1633.

[17] A. Dress, K. T. Huber, J. Koolen, V. Moulton, Blocks and cut vertices of the Buneman graph, SIAM J. Discrete Math., 4 (2011) 1902-1919.

[18] H. Hirai, Tight spans of distances and the dual fractionality of undirected multiflow problems, J. Comb. Theory, Series B, 99, 843???868, (2009)

[19] K. T. Huber, J. Koolen, and V. Moulton, The tight span of an antipodal metric space: - Part II geometrical properties, Discrete Comput. Geom., 31 (2004) 567-586.

[20] K. T. Huber, J. Koolen, and V. Moulton, On the structure of the tight span of a totally split-decomposable metric, European J. Combinatorics, $\mathbf{2 7}$ (2006) 461-479.

[21] D. Huson, D. Bryant, Application of phylogenetic networks in evolutionary studies. Mol. Biol. Evol. 23 (2006) 254-267 
[22] J. Isbell, Six theorems about metric spaces, Comment. Math.Helv. 39 (1964) 65-74.

[23] V. Klee and P. Kleinschmidt, Convex polytopes and related complexes, In: Handbook of Combinatorics, Part I, Eds. R. L. Graham, M. Grötschel, L. Lovász, Elsevier Science, North-Holland, 1999.

[24] U.Lang, Injective hulls of certain discrete metric spaces and groups, $J$. Topol. Anal. 5 (2013) 297-331.

[25] M.Pavón, Injective hulls of infinite totally split-decomposable metric spaces, arXiv:1505.07807

[26] W. A. Sutherland, Introduction to metric and topological spaces, Oxford University Press, 1983. 